\title{
Evaluación de la percepción de riesgo cardiovascular en mujeres posterior a un programa de rehabilitación cardíaca
}

\author{
María Teresa Carvallo ${ }^{1 a}$, Álvaro Maureira ${ }^{2 a}$, Marcela Lorca ${ }^{3 d}$, Eileen Hudson ${ }^{3 d}$, Yihao Wang ${ }^{4 b}$, María José Ojeda ${ }^{4 b}$, Hernán Trujillo $4 b$, \\ Sebastián Rojas ${ }^{4 b}$, Sofía Quiroz ${ }^{4 b}$, Cristine Carreño ${ }^{4 b}$, Manuel Parra ${ }^{5}$, Sebastián Calligaris ${ }^{6 c}$, Mauricio Fernández. ${ }^{6}$ \\ 1. Servicio de Medicina Física y Rehabilitación, Clínica Alemana de Santiago Universidad del Desarrollo Vitacura 5159, Vitacura, Santiago, Chile. \\ 2. Unidad de gestión Clínica Adulto, Hospital Padre Hurtado, Esperanza 2150, San Ramón, Santiago, Chile. \\ 3. Facultad de Comunicaciones, Universidad del Desarrollo, Clínica Alemana de Santiago Universidad del Desarrollo, Av. Las Condes 12.348, Lo \\ Barnechea, Santiago, Chile. \\ 4. Facultad de Medicina, Universidad del Desarrollo. Clínica Alemana de Santiago Universidad del Desarrollo. Av. Las Condes 12.348, Lo Barnechea, \\ Santiago, Chile. \\ 5. Departamento Ginecología y Obstetricia. Clínica Alemana de Santiago Universidad del Desarrollo \\ Vitacura 5159, Vitacura, Santiago, Chile. \\ 6. Departamento de Cardiología, Clínica Alemana de Santiago Universidad del Desarrollo. Vitacura 5159, Vitacura, Santiago. \\ a. Kinesiólogos. \\ b. Alumnos de la carrera de Medicina. \\ c. PhD en Ciencias Biomoleculares. \\ d. Periodistas.
}

Introducción: La rehabilitación cardíaca después de padecer un evento cardiovascular (CV) grave es un proceso en el cual el paciente establece una relación cercana con el equipo médico, brindando la oportunidad de conocer factores psicosociales que influyeron en el desenlace cardíaco y los eventuales aprendizajes de la experiencia. El objetivo de este estudio fue conocer la percepción sobre su propia salud de mujeres que participaron de un programa de rehabilitación cardíaca.

Método: Se invitó a participar a 35 mujeres de 35 a 75 años con diverso nivel educativo, ingreso familiar y situación laboral. Los datos se recolectaron por medio de entrevistas presenciales semiestructuradas. Resultados: A pesar de haber pasado por un proceso de rehabilitación posterior a un evento CV las mujeres mencionaron en forma mayoritaria al cán- cer de mama como la principal eventual causa de muerte. En relación con los factores de riesgo de CV, el más nombrado fue el estrés (57\%), aunque solo el 29\% de las entrevistadas realizó con posterioridad actividades orientadas a su manejo y control. En cuanto a las motivaciones más frecuentes para realizar cambios de hábitos, destacaron el cuidado de la familia (29\%), el vivir más (26\%) y el deseo de sentirse mejor (23\%).

Conclusión: Estos resultados sugieren la necesidad de una educación más integral en la mujer durante la rehabilitación, promoviendo no solo hábitos más saludables desde el punto de vista físico, sino también psicológico. Se sugiere integrar el manejo del estrés en los programas de prevención y rehabilitación CV. Palabras clave: salud cardiovascular; rehabilitación cardíaca; estrés; factores de riesgo; mujeres. 


\section{Perception of cardiovascular risk in women after a rehabilitation program}

Introduction: After suffering a serious cardiovascular event $(\mathrm{CV})$, cardiac rehabilitation is a process in which the patient establishes a close relationship with the medical team, providing an opportunity to learn about psychosocial factors that influence cardiac outcome and eventual learnings from the experience. The objective of this study was to learn about women's perception of their own health after participating in a cardiac rehabilitation program.

Method: 35 women from 35 to 75 years of age with varying educational level, household income and employment status were invited to participate. Data was collected through semi-structured face-to-face interviews.

Results: Women mentioned breast cancer as the leading cause of death $(60 \%)$ despite having gone through a post-CV rehabilitation process. Stress was mentioned as the main $\mathrm{CV}$ risk factor (57\%). Only $29 \%$ of responders subsequently carried out activities aimed at their management and control. The most common motivations for making changes in habits, were family care (29\%), living longer (26\%) and a desire to feel better (23\%).

Conclusion: These results suggest the need for a more comprehensive education in women during rehabilitation, promoting not only healthier habits from a physical but also from a psychological points of view. The introduction of stress management into $\mathrm{CV}$ prevention and rehabilitation programs is suggested.

Keywords: cardiovascular health; cardiac rehabilitation; stress; risk factors; women. 


\section{Introducción:}

En Chile, las enfermedades cardiovasculares son la principal causa de muerte en mujeres, de acuerdo a resultados publicados por el Instituto Nacional de Estadística en 2018, (muertes por enfermedades del sistema circulatorio, $27.3 \%$ seguida de tumores malignos, 26.3\%) ${ }^{1}$. Esta característica poblacional se puede observar globalmente, siendo similar en los Estados Unidos, en los países de la Unión Europea y también en otros países de Latinoamérica como Argentina3, Uruguay ${ }^{4}$ y México. Sin embargo, mayoritariamente la mujer percibe como la principal causa de muerte, al cáncer de mama. ${ }^{3,4,5}$

En un escenario de prevención primaria, el conocimiento de los factores de riesgo cardiovascular (FRCV) es la base para prevenir eventos $\mathrm{CV}$ y promover hábitos saludables en la mujer. Sin embargo, se ha reportado que la conciencia actual de estos en la población femenina es insuficiente ${ }^{6}$.

Por otro lado, en un escenario de prevención secundaria, es decir posterior a un evento $\mathrm{CV}$ grave la rehabilitación cardíaca tiene un efecto preventivo sobre un segundo evento CV. Se ha observado que estos programas reducen al menos en un $25 \%$ la morbilidad y la mortalidad post infarto de miocardio ${ }^{7}$. Sin embargo, se ha visto que la adherencia a estos estilos de vida saludable va disminuyendo a medida que pasa el tiempo post evento ${ }^{8}$.

Indudablemente, el padecimiento de un evento $\mathrm{CV}$ grave promueve un espacio de reflexión acerca de cómo se está viviendo hasta ese momento y crea una predisposición a adoptar hábitos de vida más saludables ${ }^{9}$. Parte de la importancia de la rehabilitación cardíaca radica en el proceso de educación y puesta en práctica de estos hábitos durante el programa. Actualmente, no existe suficiente información sobre la "nueva" percepción de la propia salud cardiovascular de la mujer después de participar de un programa de rehabilitación cardíaca. En otras palabras, ¿el programa de rehabilitación cardíaca favoreció a la toma de conciencia del riesgo asociado a la enfermedad cardiovascular, favoreciendo la incorporación de hábitos más saludables?

Por esta razón, en el presente estudio exploratorio, se decidió investigar el nivel de conocimientos y la percepción de la salud $\mathrm{CV}$, en mujeres que participaron de un programa de rehabilitación cardíaca producto de un evento cardiovascular grave.

\section{Pacientes y Método:}

\section{Diseño del estudio}

Es un estudio observacional descriptivo transversal. Se invitó a participar a mujeres que padecieron un evento $\mathrm{CV}$ y que formaron parte del programa de rehabilitación cardíaca en Clínica Alemana Santiago o en el Hospital Padre Hurtado, de las comunas de Vitacura y San Ramón, respectivamente. Previo al ingreso se les informó de objetivos, riesgos y beneficios del estudio, y la conformidad a realizar la entrevista y participar en el estudio fue documentada con un Consentimiento Informado aprobado por el Comité de Ética de la Universidad del Desarrollo. Los criterios de inclusión fueron: mujeres entre 35 a 75 años, diagnóstico de evento cardiovascular y tratamiento de rehabilitación cardíaca. Las mujeres con antecedentes de enfermedades cardíacas congénitas fueron excluidas del estudio.

\section{Recolección de los datos}

Las entrevistas se realizaron en forma presencial en ambos centros asistenciales, guiadas por un cuestionario sobre1 las variables socio-demográficas: edad, nivel educacional, ocupación, tipo de trabajo, horas diarias destinadas al trabajo, integrantes del grupo familiar, sistema de salud, estado civil e ingreso del grupo familiar ${ }^{2}$ y el conocimiento y percepción de la salud cardiovascular femenina. El listado de las preguntas se describe en el Anexo 1. En la formulación de algunas preguntas y en la categorización de estas se consideró como referencia el estudio de Mosca y colaboradores ${ }^{10}$.

La muestra fue intencionada y razonada, evaluándose al final de dichas entrevistas la necesidad de incluir a nuevas participantes, hasta que se logró el criterio de saturación de contenidos temáticos ${ }^{11}$.

La entrevista fue grabada como audio y luego transcrita. Los audios fueron conservados hasta la finalización del estudio. Las respuestas a las preguntas fueron registradas en hojas de cálculo Microsoft Excel® para su posterior análisis. La información socio-demográfica, así como la relativa a la percepción de salud cardiovascular de cada paciente, se codificó para asegurar el anonimato de las respuestas. Los datos fueron analizados por medio de tablas de contingencia, con las cuales se estableció la frecuencia de cada variable nominal o categórica. Los datos fueron visualizados y analizados mediante el programa Prism (GraphPad, Inc.).

\section{Resultados:}

Se entrevistó a 35 mujeres con antecedente de haber presentado un infarto agudo al miocardio por dos motivos principales: disección espontánea o enfermedad ateromatosa de las arterias coronarias, en las pacientes más 
jóvenes y añosas, respectivamente.

El 69\% de mujeres entrevistadas se ubicó en el rango de edad de 45 a 64 años. Con respecto al estado civil, el $50 \%$ se reportó como casada y el resto se distribuyó en las tres categorías restantes: divorciada/separada, viuda o soltera (Tabla 1).

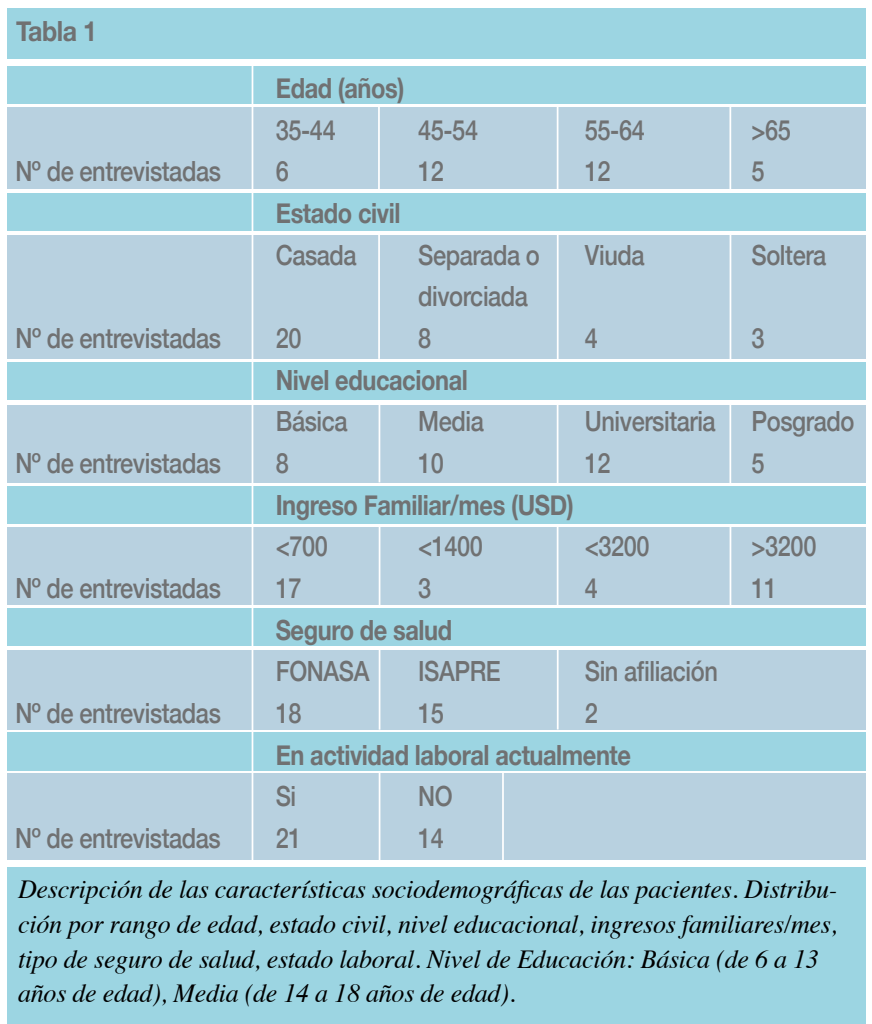

En las entrevistadas, el nivel educacional se distribuye de una manera equilibrada, desde la educación básica incompleta hasta estudios de posgrado. La educación escolar en Chile se divide en tres períodos: básica (de los 6 a 13 años), media (de los 14 a 18 años) y superior (incluyendo a la universitaria) ${ }^{12}$. Por otra parte, el ingreso familiar, convertido a dólares estadounidenses (USD), fue categorizado como $<700$ y $>700$ USD, lo cual refleja dos grupos: uno afiliado al programa de seguros de salud del Gobierno de Chile (FONASA) y otro a instituciones privadas (ISAPRES). En el año 2019, el salario mínimo fue equivalente a 420 dólares de acuerdo a la última ley de presupuesto aprobada por el Senado de la República Chilena ${ }^{13}$. El 60\% de las entrevistadas desarrolla una actividad laboral, ya sea de carácter independiente o dependiente (Tabla 1).

Frente a la pregunta de qué problema de salud considera más relevante para la mujer en Chile, las respuestas son las ECV y el cáncer, seguido en tercer lugar por el estrés, la obesidad y las enfermedades mentales incluyendo depresión y ansiedad (Figura 1A) ${ }^{14}$. Frente a la pregunta de cual consideraban como la primera causa de muerte en mujeres, el $60 \%$ de las entrevistadas señaló a la enfermedad oncológica, principalmente, el cáncer de mama (Figura 1B), seguido de las ECV y en menor proporción, el femicidio.

En la descripción de los síntomas del infarto agudo de miocardio, si bien hubo aspectos en común en el testimonio, la secuencia, duración y variedad de los síntomas,

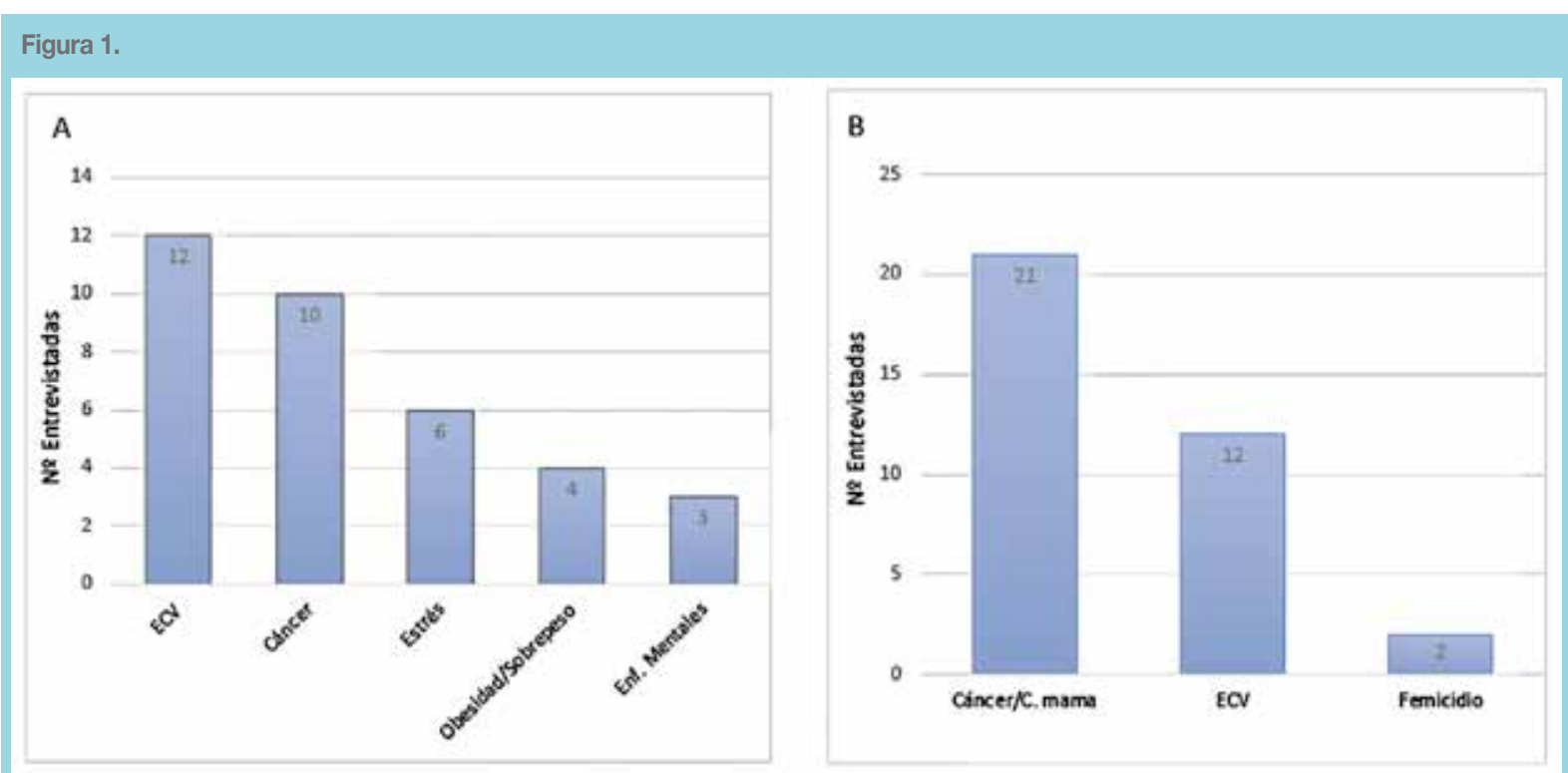

Problemas de salud más relevantes (A); Principales causas de muerte (B); enfermedades cardiovasculares (ECV). 


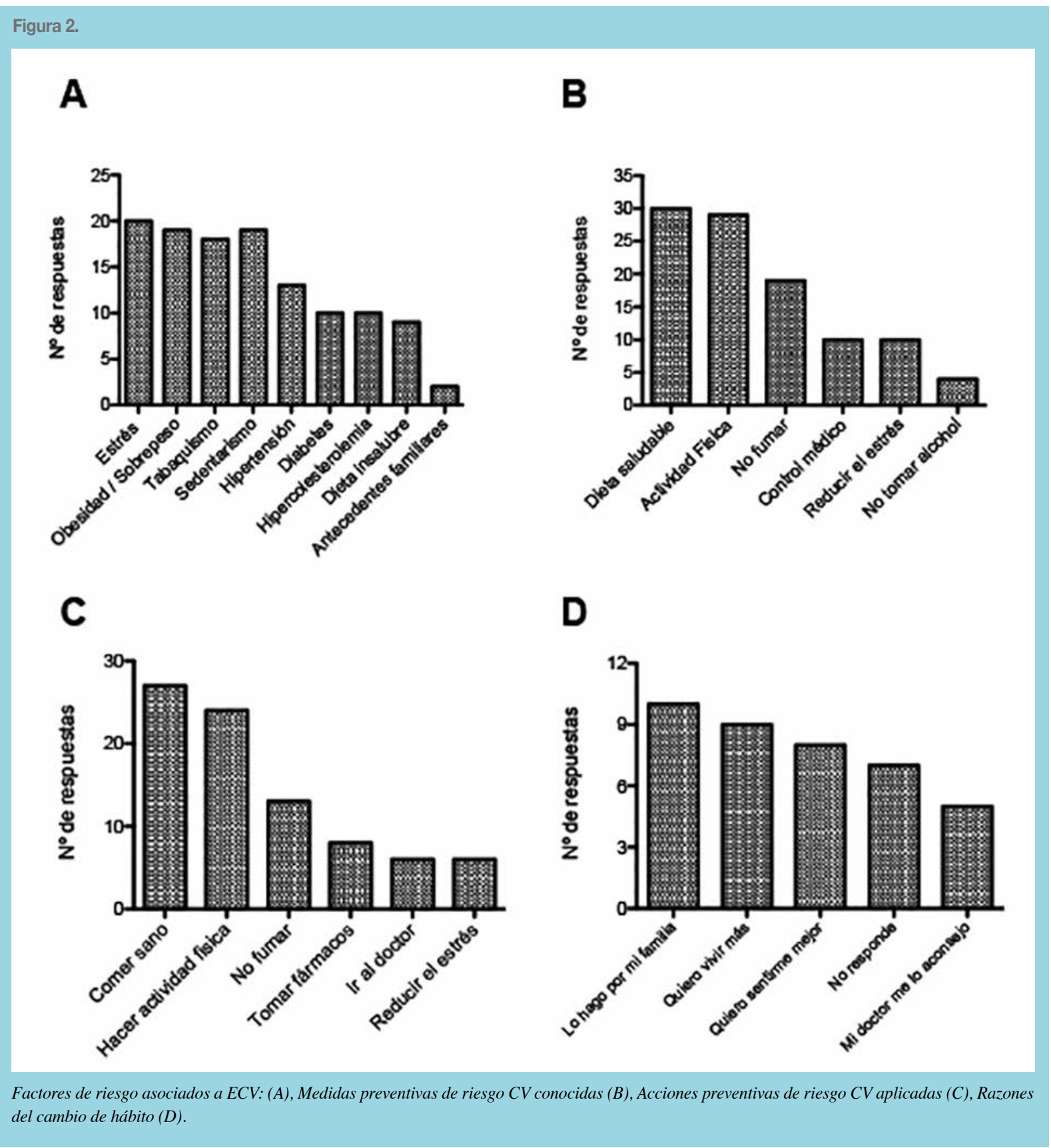

fue particular en cada mujer. En cambio, sí se encontró casi total coincidencia en la confusión de la percepción del propio estado de salud, en el momento que estaba cursando el evento cardiovascular. De hecho, el $80 \%$ de las entrevistadas no sabía que tenía riesgo de padecer un evento cardiovascular.

En relación con el conocimiento de los FRCV, las variables más frecuentemente reconocidas fueron el estrés, la obesidad, el tabaquismo y el sedentarismo, seguido de un segundo grupo: hipertensión arterial, diabetes, hiperco- lesterolemia y dieta no saludable (Figura 2A). Por otra parte, se observó una correspondencia entre los FRCV y las medidas preventivas que conocían: sedentarismo/actividad física, obesidad/dieta saludable y tabaquismo/no fumar. Sin embargo, la reducción del estrés fue considerada en menor proporción entre las mujeres entrevistadas (Figura 2B). De hecho, las medidas preventivas mayormente adoptadas fueron: comer sano, realizar actividad física y no fumar, siendo la respuesta: "reducir el estrés", una de las menos frecuentes (Figura 2C). 
Con respecto a los motivos que promovieron los cambios de hábitos, la intención de cuidar la familia, querer vivir más y sentirse mejor, fueron las respuestas más frecuentes (Figura 3D).

\section{Discusión:}

Sorprendentemente, en promedio dos años después de haber padecido un infarto al miocardio, la mayoría de las mujeres entrevistadas en este estudio considera que la primera causa de muerte en la mujer es el cáncer (principalmente, el cáncer de mama).

Las características sociodemográficas describen un grupo de mujeres con un nivel educacional, estado laboral e ingreso familiar diverso pero equilibrado entre las distintas categorías, lo cual sugiere que la falta de conocimiento sobre su propia salud cardiovascular es transversal en la población femenina chilena.

El FRCV más citado fue el estrés, lo cual concuerda con la percepción personal que una situación de estrés aguda (ej.: discusión con un familiar) o crónica (ej.: gestión de actividades familiares y laborales) fue el desencadenante del evento cardiovascular padecido, independientemente del grado de riesgo CV. No obstante, dentro de las medidas preventivas conocidas por las entrevistadas, la opción "reducir el estrés" fue poco frecuente. De hecho, una minoría de mujeres declaró haber realizado medidas para mitigar su estrés. Blumenthal y colaboradores demostraron que la incorporación de un entrenamiento para manejar el estrés durante la rehabilitación cardíaca (tanto de hombres como mujeres), mejora no solo su estado anímico, sino el estado de salud CV de los pacientes a tres años promedio de seguimiento ${ }^{15}$.

La mayoría de las mujeres manifiesta no tener problemas para adoptar las medidas que consideran saludables como: dejar de fumar, hacer actividad física y adoptar una dieta saludable (reducida en grasa y azúcares), siendo el "motor" de cambio el bienestar personal y el de su propio entorno (ej.: cuidado de hijos o nietos). Sin embargo, esta última motivación se ha descrito también como un factor psicosocial de género que incide en el estrés producto de la sobre exigencia y la sobrecarga de las mujeres, tanto en el hogar, como en el trabajo ${ }^{16}$.

En conclusión, el programa de educación dentro de la rehabilitación cardíaca post evento cardiovascular, brindó a las mujeres una mejor comprensión de cómo modificar su condición física pero observamos una carencia en el apoyo psicológico que contribuya a manejar el estrés para poder reducir aún más la probabilidad de reincidencia de un evento cardiovascular.

\section{Agradecimientos:}

Agradecemos la valiosa colaboración de EU Catalina Rosales y EU Constanza Honorato en la coordinación de las reuniones con las pacientes.

\section{Anexo 1}

Cuestionario utilizado para las entrevistas

Datos socio-demográficos

\section{1. ¿Cuál es su edad?}

\section{2. ¿Cuál es su nivel educacional?}

a. Educación básica

b. Educación media

c. Educación universitaria

d. Posgrado

3. ¿Cuál de las siguientes alternativas describe mejor su situación? (se refiere a trabajo remunerado)

a. Actualmente estoy trabajando

b. No estoy trabajando actualmente

\section{4. ¿A cuál sistema de salud pertenece Ud.?}

a. Sistema público FONASA

b. ISAPRE

c. Ninguno (particular)

7. ¿Cuál es su estado civil o conyugal actual?

a. Casada

b. Viuda

c. Divorciada

d. Separada (casada legalmente)

e. Soltera, nunca se ha casado

Salud cardiovascular

1. ¿Cuál piensa que es el problema de salud más importante de la mujer chilena? 2. ¿Cuál cree que es la causa más frecuente de muerte
en la mujer?

3. ¿Con qué factores de riesgo asocia a las enfermedades cardiovasculares?

4. ¿Qué medidas de prevención de riesgo cardiovascular conoce? 
5. ¿Realiza acciones preventivas para proteger su salud cardiovascular? ¿Cuáles? ¿Ha tenido dificultades para incorporar dichas medidas? ¿Por qué?

6. ¿Sabía usted que tenía riesgo de sufrir un evento cardiovascular?
7. Luego de haber vivido este evento cardiovascular, ¿Cómo ha cambiado su vida? ¿La experiencia la motivó a reflexionar sobre cómo estaba conduciendo su vida? ¿Realizó algún cambio que encuentra beneficioso para usted? ¿Ha tenido apoyo familiar o de otras personas?

\section{Referencias}

1. ANUARIO DE ESTADISTICAS VITALES 2018. Instituto Nacional de Estadística, Chile.

3. PRAMPARO P. ¿Falta de concientización de las mujeres sobre la enfermedad cardiovascular o información inadecuada e insuficiente? Revista Argentina de Cardiología 2013; 81: 301-303.

4. COMITÉ DE CARDIOPATÍA EN LA MUJER SUDC. Mujeres uruguayas tienen percepción falsa de su principal causa de muerte. El Observador, 2014.

5. JUÁREZ-HERRERA L, CASTRO-VÁSQUEZ, M. Y RUÍZ-CANTERO, M. Análisis con perspectiva de género sobre perceopción y prácticas en enfermedad coronaria en mujeres en el norte de México. Salud Pública de México 2016; 58: 428-433.

6. KUNSTMANN S, LIRA, MT., ICAZA, G., ÑUÑEZ, L. Y GRAZIA, R. Estratificación de riesgo cardiovascular en la población chiilena. Revista Médica Clínica Las Condes 2012.

7. MAMPUYA WM. Cardiac rehabilitation past, present and future: an overview. Cardiovasc Diagn Ther. 2012 Mar;2(1):38-49. doi:10.3978/j.issn.2223-3652.2012.01.02. PMID: 24282695; PMCID: PMC3839175

8 SANTIAGO DE ARAÚJO PIO C, CHAVES GSS, DAVIES $\mathrm{P}$, TAYLOR RS, GRACE SL. Interventions to promote patient utilisation of cardiac rehabilitation. Cochrane Database of Systematic Reviews 2019, Issue 2. Art. No.: CD007131. DOI: 10.1002/14651858.CD007131.pub4

9 STEPTOE A, KIVIMÄKI M. Stress and cardiovascular disease. Nat Rev Cardiol. 2012 Apr 3;9(6):360-70. doi: 10.1038/ nrcardio.2012.45. PMID: 22473079.

10. MOSCA L, HAMMOND G, MOCHARI-GREENBERGER $\mathrm{H}$, et al. Fifteen-year trends in awareness of heart disease in women: results of a 2012 American Heart Association national survey. Circulation 2013; 127: 1254-1263, e1251-1229. Comparative Study Research Support, N.I.H., Extramural 2013/02/23. DOI: 10.1161/CIR.0b013e318287cf2f.

11. MARTINEZ-SALGADO C. El muestreo en investigación cualitatia. Principios básicos y algunas controversias. Ciencia \& Saúde coletiva 2012; 17: 613-619.

12. MINUDEC CDE. ¿Hacia dónde avanza el sistema educativo en Chile? Serie Evidencias 2017; 37.

13. CHILENA SDLR. Ley de salario mínimo, http://www.senado.cl (2019).

14. ENCUESTA NACIONAL DE SALUD (2016-2017) Ministerio de Salud (MINSAL) Gobierno de Chile.

15. BLUMENTHAL JA, SHERWOOD A, SMITH PJ, et al. Enhancing Cardiac Rehabilitation With Stress Management Training: A Randomized, Clinical Efficacy Trial. Circulation 2016; 133: 1341-1350. Comparative Study Randomized Controlled Trial Research Support, N.I.H., Extramural 2016/04/06. DOI: 10.1161/CIRCULATIONAHA.115.018926.

16. FERNANDEZ-MORENO, Sara Yaneth. Heridos corazones. Vulnerabilidad coronaria en varones y mujeres, de Débora Tajer, Buenos Aires, Paidós, 2009. Rev. Gerenc. Polit. Salud [online]. 2010, vol.9, n.18, pp.166-168. ISSN 1657-7027. 